Original Article (short paper)

\title{
Time to task failure of the contralateral untrained limb after high load-low repetition eccentric and low load-high repetition resistance training
}

\author{
Nosratollah Hedayatpour \\ Ali Golestani \\ Zahra Izanloo \\ University of Bojnord, Bojnord, Iran \\ Ali Sepehri \\ Azad University of Bojnord, Bojnord, Iran \\ Maryam Kamali \\ University of Bojnord, Bojnord, Iran
}

\begin{abstract}
Aims: Cross-training is the process whereby training of one limb gives rise to enhancements in the performance of the opposite, untrained limb and may be dependent on type of muscle contractions performed. The aim of this study was to investigate whether unilateral resistance training using eccentric contraction is more effective than concentric resistance training to improve time to task failure in the contralateral untrained limb. Methods: Subjects completed 12 weeks of resistance training consisting of 36 sessions, using unilateral leg exercise. Sustained isometric knee extension performed at $50 \%$ of maxmal force until task failure for the contralateral untrained leg. Surface electromyography (EMG) signals were simultaneously recorded from contralateral untrained quadriceps (vastus medialis, rectus femoris, and vastus lateralis). Results: Time to task failure of the contralateral untrained leg and associated EMG activities significantly increased after 12 weeks of unilateral resistance training $(p<0.05)$. However, percent increase in time to task failure and EMG amplitude after eccentric resistance training was significantly higher than concentric resistance training $(\mathrm{p}<0.05)$. Conclusion: This study concluded that unilateral eccentric resistance training is superior to concentric resistance training to increase time to task failure in the contralateral untrained limb.
\end{abstract}

Keywords: EMG, contralateral, quadriceps, task failure, eccentric;

\section{Introduction}

Task failure during a sustained submaximal task is thought to be largely related to impaired motor drive $e^{1,2,3}$ as evidenced by twitch interpolation and transcranial magnetic stimulation (TMS) technique for the upper and lower limb muscles ${ }^{4,5,6}$. Previous studies have also shown that time to task failure of sustained contraction was prolonged following resistance training, most likely due to neural adaptations at level of central nerve system ${ }^{8}$.

Cross-training is the process whereby training of one limb gives rise to enhancements in the performance of the opposite, untrained limb ${ }^{9}$. Numerous studies have investigated cross training following strength training. For example, Adamson et al. ${ }^{10}$ reported that unilateral arm strength training improves contralateral peak force and rate of force development .Accordingly, cross-training effect has been observed in different muscles groups after different mode of exercise training and at different levels of task complexity ${ }^{9,11}$. It has been reported that crosstraining process is primarily caused by adaptations in the central nervous system CNS) for the untrained limb following exercise training ${ }^{12,13,14}$. However, neural adaptions to resistance training are dependent on type of muscle contractions performed ${ }^{15,16,17}$. For example, previous studies reported greater cortical activities during eccentric task ${ }^{18}$, and a higher motor unit discharge rates after eccentric exercise as compared to concentric exercise ${ }^{19}$. Moreover, improvements in maximal muscle force, peak torque and muscle hypertrophy after eccentric training was significantly larger than those observed after concentric training ${ }^{16,20}$. Since greater maximum muscle force and neural activity can be developed during maximal eccentric exercise compared to concentric exercise, heavy-resistance training using eccentric muscle actions may be most effective for increasing muscle ability to maintain a submaximal force. These adaptations may also be transferred to the opposite homologous limb as evidenced by neural plasticity of motor pathways projecting to the contralateral untrained limb and or/trained limb $\mathrm{in}^{21,22}$.

However, there are no studies that have investigated time to task failure in homologous muscle in the contralateral limb after unilateral eccentric exercise training. This knowledge maybe useful for exercise training and/or rehabilitation program. Therefore, the purpose of this study was to compare time to task failure in homologous muscle of the opposite limb after 12 weeks of unilateral eccentric and concentric exercise training. Surface electromyography signals (EMG) were recorded from the contralateral untrained quadriceps muscles during submaximal contraction sustained at $50 \%$ of maximal voluntary contraction (MVC) until task failure. 


\section{Materials and methods}

Subjects: Thirty healthy, male subjects (age, mean \pm SD, $24.2 \pm 1.9$ yr., body mass $71.3 \pm 10.5 \mathrm{~kg}$, height $1.75 \pm 0.05$ $\mathrm{m}$ ) with no history of knee injury or trauma participated to the study. Subjects were randomly divided into two training group: high load-low repetition eccentric group $(n=15)$ and low load-high repetition concentric group $(\mathrm{n}=15)$. All subjects were right leg dominant and were not involved in regular exercise of their knee extensor muscles for at least 6 months before the experiment. The study was conducted in accordance with the Declaration of Helsinki, approved by the Local Ethics Committee, and written informed consent was obtained from all subjects prior to inclusion.

Exercise training. All subjects completed 12 weeks of resistance training using unilateral leg press eccentric and concentric action. Eccentric training group $(3$ sets $\times 5$ reps using $120 \% 1 \mathrm{RM})$. Concentric training group ( 3 sets $\times 10$ reps using $60 \% 1 \mathrm{RM})$. Three minutes of rest was considered between sets. Unilateral leg press exercise was performed through $90^{\circ}$ to $180^{\circ}$ of knee extension $\left(180^{\circ}=\right.$ full extension $)$ using a weight-training machine (Universal Gym). Timing of the lifting, lowering and lockout phases of the exercise was established using a metronome. The metronome emitted an audible stimulus at a frequency of $1 \mathrm{~Hz}$. Subjects were asked to maintain a cadence of 2 during the lifting phase, 1 during the lockout and 3 during the lowering phase, in time with the metronome. 1-RM was evaluated for each subject every week and the weights were adjusted accordingly. Because the weights were adjusted every week, the number of repetitions was also adjusted so that the total weight lifted by each subjects could be equated. The workloads were determined for the subject based on their initial one repetition maximum (1-RM). One repetition maximum is defined as the heaviest load that can be moved over a specific range of motion, one time, and with correct performance ${ }^{23}$. Each subject was required to be able to lift his maximum load in a smooth, controlled motion. In the eccentric contraction, a helper lifted the load to the stating position $\left(170^{\circ}-180^{\circ}\right.$ knee angle: $180^{\circ}=$ full knee extension), and the subject lowered the load to the finish position $\left(90^{\circ}\right.$ knee angle) in a controlled manner. The subjects were encouraged to resist against the load during eccentric contraction. Use of helper permitted subjects to do multiple repetitions against relatively high loads and delayed the onset of fatigue by eliminating concentric contraction.

Muscle function: The subject sat comfortably on a chair fixed with a belt at the hip with the right knee $90^{\circ}$ flexed. A strap connected by a chain to a load cell was attached to the ankle to measure knee extension isometric force. The subject performed three maximal voluntary contractions (MVC) separated by 2-min rest. During each MVC contraction, verbal encouragement was provided. Participants also performed isometric knee extension contraction at 50\% MVC sustained until task failure in random order, with the participant in the same position as in the maximal voluntary contractions. The submaximal force was relative to the highest MVC measured on the same day of the test. Task failure was defined as a drop in torque $>5 \% \mathrm{MVC}$ for more than $5 \mathrm{~s}$ after strong verbal encouragement to the subject to maintain the target torque. Surface EMG signals were simultaneously recorded from vastus medialis, rectus femoris and vastus lateralis muscles during isometric knee extension contraction at 50\% MVC, which was sustained until task failure.

\section{Surface EMG recordings}

Three pairs of circular $(\mathrm{Ag}-\mathrm{AgCl}$ surface electrodes $\mathrm{Ambu}$ Neuroline, Ambu A/S, Ballerup, Denmark; conductive area = $28 \mathrm{~mm} 2$ ) were placed in bipolar configuration (interelectrode distance $=2 \mathrm{~cm}$ ) over the quadriceps femoris muscle. Electrodes were placed over the quadriceps components at $10 \%$ of the distance between medial border, superior border and lateral border of the patella (for vastus medialis (VM), rectus femoris (RF) and vastus lateralis (VL) respectively) and anterior superior iliac spine $^{24}$. Before electrode placement, the skin was shaved and lightly abraded at the selected locations. Surface EMG signals were amplified bipolarly (EMG amplifier, EMG-16, LISiN - OT Bioelettronica, Torino, Italy; bandwidth 10-500 Hz), sampled at $2048 \mathrm{~Hz}$, and stored after $12 \mathrm{bit} \mathrm{A} / \mathrm{D}$ conversion.

\section{Signal analysis}

Average rectified value (ARV) was estimated from the EMG signals for epochs of $1 \mathrm{~s}$. The values obtained from 1-s-long epochs in intervals of $10 \%$ of the time to task failure were averaged to obtain one representative value for each $10 \%$ interval. This was done to compare subjects that had different times to task failure. The percent change in EMG ARV over time was calculated by subtracting the final value (last $10 \%$ interval) from the initial value (first $10 \%$ interval) of EMG ARV and dividing by the initial value. To compare changes across sessions, differences in ARV for post training with respect to pre training were calculated.

\section{Statistical Analysis}

One-ways repeated-measures ANOVA was applied to change in MVCs and time to task failure from pre - to post-training session with training group (eccentric and concentric) as dependent factor. A two-ways ANOVA was also applied to the percent change in EMG over sustained contraction with testing session (pre - post training) and training group (eccentric and concentric ) as dependent factors. Pairwise comparisons were performed with the Student-Newman-Keuls post hoc test when ANOVA was significant.

\section{Results}

Maximal voluntary contraction was significantly increased after eccentric and concentric resistance training $(\mathrm{P}<0.0001, \mathrm{~F}=34.4)$. 
Percent increase in MVC after eccentric training was significantly larger than the concentric training $(\mathrm{P}<0.001)$. Figure 1.

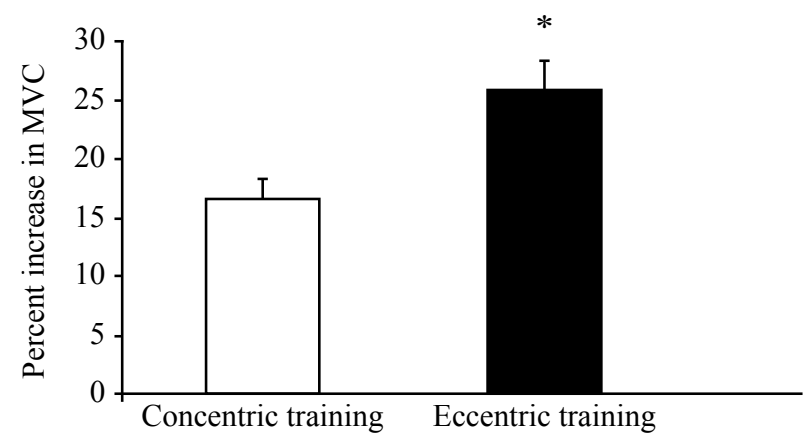

Figure 1 - Percent increase in maximal voluntary contraction (MVC) of the contralateral untrained quadriceps muscle (mean $\pm \mathrm{SE}, \mathrm{s}$, $\mathrm{n}=15$ ) after 12 weeks resistance training. Percent increase in MVC after eccentric training is significantly larger than concentric training. $* p \leq 0.05$.

Time to task failure increased after eccentric and concentric resistance training $(\mathrm{F}=124.9, \mathrm{P}<0.0001)$. Percent increase in time to task failure depend on training mode with a larger increase identified for eccentric group compared to concentric group $(\mathrm{P}<0.05)$ Figure 2.

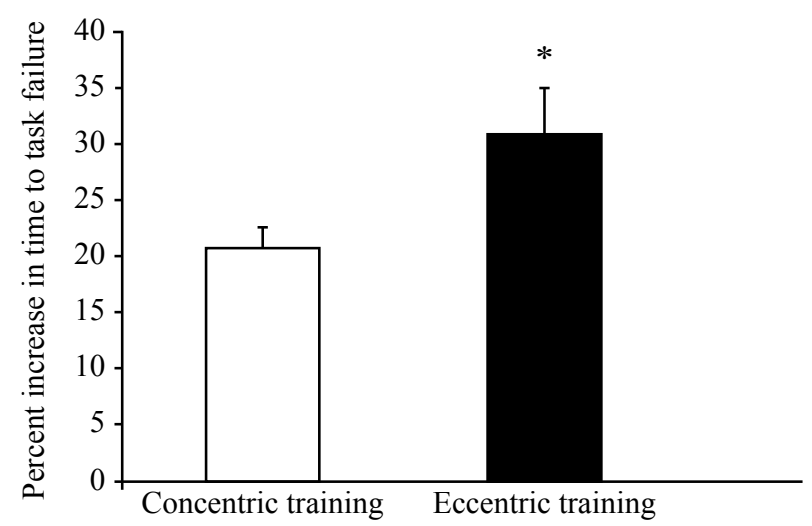

Figure 2 - Percent increase in time to task failure of the contralateral untrained leg (mean $\pm \mathrm{SE}, \mathrm{s}, \mathrm{n}=15$ ) during sustained contraction at $50 \%$ MVC after 12 weeks resistance training. Percent increase in time to task failure after eccentric training is significantly larger than concentric training. ${ }^{*} \mathrm{p} \leq 0.05$.

ARV of EMG significantly increased over time during sustained contraction at 50\% MVC $(\mathrm{F}=17.6, \mathrm{P}<0.001)$. EMG ARV in the last $10 \%$ interval of the time to task failure was significantly larger than the initial 10\% interval $(\mathrm{P}<0.05)$.

Moreover, the ARV of EMG during post training sustained contractions was significantly larger than pre training condition $(\mathrm{F}=12.6, \mathrm{P}<0.001)$.

Percent increase in EMG ARV (percent increase from the initial $10 \%$ interval to the last $10 \%$ interval of the time to task failure) depend on training mode with a larger increase identified for eccentric group compared to concentric group $(\mathrm{P}<0.05)$. Figure 3

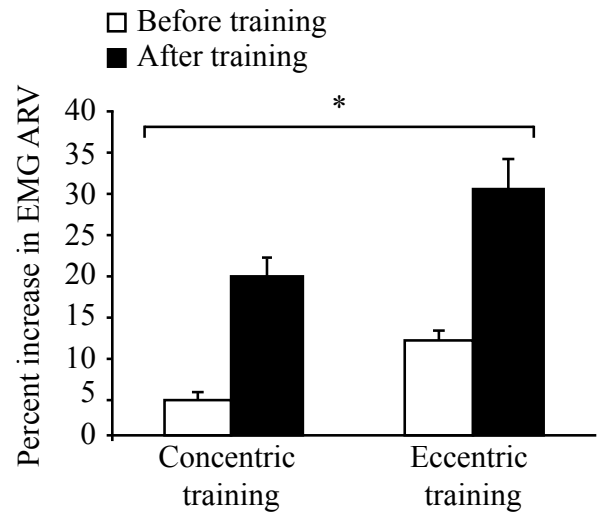

Figure 3 - Percent increase in average rectified value of EMG (percent increase from the initial $10 \%$ interval to the last $10 \%$ interval of time to task failure) for the untrained leg (average for the VM, RF and VL muscles) during sustained isometric knee extension at 50\% of MVC measured before and after 12 weeks resistance training. ${ }^{*} \mathrm{p} \leq 0.05$.

\section{Discussion}

The result of this study demonstrated that time to task failure rate of increase in the contralateral untrained leg following 12 weeks high intensity-low volume eccentric exercise was significantly larger than those observed after low intensity-high volume concentric exercise. Moreover the EMG amplitude of quadriceps muscle in the contralateral untrained leg significantly increased during sustained contraction after resistance training, with a higher increase obtained after high intensity-low volume eccentric exercise as compared with low intensity-high volume concentric exercise.

\section{Sustained contraction of the untrained leg before unilateral resistance training}

EMG amplitude of the quadriceps muscle in the untrained leg progressively increased over time during submaximal sustained contraction at $50 \% \mathrm{MVC}$ to maintain force output. Previous studies have also reported, a longer duration of a submaximal task was associated with an increase in EMG activity over time ${ }^{25}$. These studies suggested that the increased EMG amplitude is related to the enhanced neural drive from higher motor center to muscle fibers to increase motor unit recruitment and/or discharge rate required to compensate for contractile failure caused by fatigue ${ }^{25}$.

\section{Sustained contraction of the untrained leg after unilateral resistance training}

Time to task failure of the contralateral untrained leg was significantly prolonged after both eccentric and concentric resistance training. Accordingly, The EMG amplitude of quadriceps muscle in the contralateral untrained leg significantly increased during post training sustained contraction as compared with pre training. The current study is the first to assess time to task failure in the contralateral untrained limb following unilateral eccentric 
and concentric resistance training. In this study, unilateral high intensity-low volume eccentric exercise resulted in a greater increase in time to task failure and associated EMG activities as compared with low intensity - high volume concentric exercise. These results are in agreement with previous work suggesting cross-training effect in different muscles groups after different mode of exercise training and at different levels of task complexity ${ }^{11}$.

For example, maximal muscle force increased at an average rate of $7 \%$ to $8 \%$ for the contralateral untrained homologous muscle group after 4 to 12 weeks of unilateral training 9 . Furthermore, the increased maximal force in the contralateral untrained limb after eccentric training was significantly larger than concentric training ${ }^{11,26}$, which is in agreement with our findings. These lines of evidence suggest that unilateral eccentric exercise of a specific limb result in greater cross training effects in the untrained contralateral limb as compared with concentric and/or isometric exercise. Accordingly, we also demonstrated high intensity eccentric exercise is more effective than concentric exercise to increase time to task failure in the contralateral untrained leg.

The superiority of eccentric exercise to increase time to task failure and /or maximal force in contralateral untrained limb may be explained by several potential mechanisms.

Eccentric contraction is characterized by the preferential recruitment of fast twitch motor units and higher cortical activation compared to concentric contractions ${ }^{27,28}$. During a sustained contraction, fast twitch motor units are recruited later than the slow ones ${ }^{29}$, and therefore help to sustain the force output. Repeated recruitment of fast twitch motor units during eccentric training reduce their recruitment threshold ${ }^{30}$, and facilities their contribution to sustain force output during a fatiguing contraction. A longer time to task failure observed after eccentric training may also be related to higher cortical activation during eccentric exercise ${ }^{18}$, which in turn result in an enhanced neural drive in the corticospinal pathways to sustain muscle force. Moreover, eccentric training uniquely modulates neural regulatory pathways involved in the excitation and inhibition process and to a greater extent than concentric training ${ }^{20,31,32}$. The opposite hemisphere may access these modified circuits during voluntary contraction of the opposite untrained limb and enhance the ability of muscle to maintain force output ${ }^{11}$.

\section{Conclusion}

Unilateral high intensity eccentric exercise resulted in higher increase in time to task failure of the contralateral untrained leg as compared to concentric exercise. The superiority of eccentric exercise in increasing time to task failure of the contralateral untrained limb may be related to the unique neural plasticity of motor pathways projecting to the contralateral untrained limb. This knowledge may be useful to understand the mechanisms underlying cross training which in turn contribute to more effective use of resistance training protocols to improve the recovery of patients with movement disorders that predominantly affect one side of the body.

\section{References}

1. Place N, Bruton JD, Westerblad H. Mechanisms of fatigue induced by isometric contractions in exercising humans and in mouse isolated single muscle fibres. Clin Exp Pharmacol Physiol. 2009; 36(3): 334-9.

2. Hedayatpour N, Arendt-Nielsen L, Farina D. Motor unit conduction velocity during sustained contraction of the vastus medialis muscle. Exp Brain Res. 2007; 180: 509-16.

3. Hedayatpour N, Arendt-Nielsen L, Farina D. Non-uniform electromyographic activity during fatigue and recovery of the vastus medialis and lateralis muscles. J Electromyogr Kinesiol. 2008; 18:390-6.

4. Löscher WN, Cresswell AG, Thorstensson A. Central fatigue during a long-lasting submaximal contraction of the triceps surae. Exp Brain Res. 1996; 108: 305-14.

5. Löscher WN, Cresswell AG, Thorstensson A. Excitatory drive to the alpha-motoneuron pool during a fatiguing submaximal contraction in man. J Physiol. 1996; 491: 271-80.

6. Zijdewind I, Zwarts MJ, Kernell D. Influence of a voluntary fatigue test on the contralateral homologous muscle in humans? Neurosci Lett. 1998; 253: 41-4.

7. Ljubisavljević M, Milanović S, Radovanović S, Vukcević I, Kostić V, Anastasijević R. Central changes in muscle fatigue during sustained submaximal isometric voluntary contraction as revealed by transcranial magnetic stimulation. Electroencephalogr Clin Neurophysiol. 1996; 101: 281-8.

8. Vila-Chã C, Falla D, Farina D. Motor unit behavior during submaximal contractions following six weeks of either endurance or strength training. J Appl Physiol. 2010; 109: 1455-66.

9. Carroll TJ, Herbert RD, Munn J, Lee M, Gandevia SC. Contralateral effects of unilateral strength training, evidence and possible mechanisms. J Appl Physiol. 2006; 101: 1514-22.

10. Adamson M, Macquaide N, Helgerud J, Hoff J, Kemi OJ. Unilateral arm strength training improves contralateral peak force and rate of force development. Eur J Appl Physiol. 2008; 103: 553-9.

11. Hortobágyi T, Lambert NJ, Hill P. Greater cross education following training with muscle lengthening than shortening. Med Sci Sports Exerc. 1997; 29: 107-12.

12. Bezerra P, Zhou S, Crowley Z, Brooks L, Hooper A. Effects of unilateral electromyostimulation superimposed on voluntary training on strength and cross-sectional area. Muscle Nerve. 2009; 40: 430-7.

13. Dragert K, Zehr EP. Bilateral neuromuscular plasticity from unilateral training of the ankle dorsiflexors. Exp Brain Res. 2011; 208: 217-27.

14. Everaert DG, Thompson A.K, Chong S.L, Stein R.B. Functional electrical stimulation for foot drop strengthen corticospinal connections? Neurorehabil Neural Repair. 2010; 24: 168-77.

15. Patten C, Kamen G, Rowland DM. Adaptations in maximal motor unit discharge rate to strength training in young and older adults. Muscle Nerve. 2001; 24: 542-50.

16. Farthing JP, Chilibeck PD. The effects of eccentric and concentric training at different velocities on muscle hypertrophy. Eur J Appl Physiol. 2003; 89(6): 578-86. 
17. Hedayatpour N, Falla D. Physiological and Neural Adaptations to Eccentric Exercise, Mechanisms and Considerations for Training. Biomed Res Int. 2015, 193741.

18. Fang Y, Siemionow V, Sahgal V, Xiong F, Yue GH. Greater movement-related cortical potential during human eccentric versus concentric muscle contractions. J Neurophysiol. 2001; 86(4): 1764-72.

19. Dartnall TJ, Rogasch NC, Nordstrom MA, Semmler JG. Eccentric muscle damage has variable effects on motor unit recruitment thresholds and discharge patterns in elbow flexor muscles. J Neurophysiol. 2009; 102(1): 413-23.

20. Kaminski TW, Wabbersen CV, Murphy RM. Concentric versus enhanced eccentric hamstring strength training: clinical implications. J Athl Train. 1998; 33(3):216-21.

21. Sotgiu ML, Brambilla M, Valente M, Biella GE. Contralateral input modulates the excitability of dorsal horn neurons involved in noxious signal processes. Potential role in neuronal sensitization. Somatosens Mot Re. 2004; 21: 211-5.

22. Hortobágyi T, Richardson S.P, Lomarev M, Shamim E.A, Meunier $\mathrm{S}$, Russmann H, et al. Interhemispheric plasticity in humans. Med. Sci. Sports Exerc. 2011; 43: 1188-1199.

23. Pereira M, Gomes P. Muscular strength and endurance tests reliability and prediction of one repetition maximum. Rev Bras Med Esporte. 2003; 9: 325-335.

24. Hedayatpour N, Falla D, Arendt-Nielsen L, Farina D. Sensory and electromyographic mapping during delayed-onset muscle soreness. Med Sci Sports Exerc 2008; 40: 326-34.

25. Kirsch RF, Rymer WZ. Neural compensation for fatigue-induced changes in muscle stiffness during perturbations of elbow angle in human. J Neurophysiol. 68, 449-70.

26. Seger J.Y, Thorstensson A. (2005). Effects of eccentric versus concentric training on thigh muscle strength and EMG. Int $\mathrm{J}$ Sports Me 1992; 26(1): 45-52.
27. Moritani T, Muramatsu S, Muro M. Activity of motor units during concentric and eccentric contractions. Am J Phys Med. 1987; 66(6): 338-50.

28. Nakazawa K, Kawakami Y, Fukunaga T, Yano H, Miyashita M. Differences in activation patterns in elbow flexor muscles during isometric, concentric and eccentric contractions. Eur J Appl Physiol Occup Physiol 1993; 66(3): 214-20.

29. Mendell LM. The size principle: a rule describing the recruitment of motoneurons. J Neurophysiol. 2005; 93(6): 3024-6.

30. Duchateau J, Semmler JG, Enoka RM. Training adaptations in the behavior of human motor units. J Appl Physiol. 2006; 101(6):1766-75

31. Aagaard P, Simonsen EB, Andersen JL, Magnusson SP, HalkjaerKristensen J, Dyhre-Poulsen P. Neural inhibition during maximal eccentric and concentric quadriceps contraction: effects of resistance training. J Appl Physiol. 2000; 8: 2249-57

32. Bawa P. Neural control of motor output: can training change it? Exerc Sport Sci Rev. 2002; 30(2): 59-63.

\section{Corresponding author}

Nosratollah Hedayatpour, Ph.D

Center for Biomechanic and Motor Control (BMC), Department of Physical Education and Sport Science, University of Bojnord, Bojnord, Iran

Email: n.hedayatpour@ub.ac.ir

Manuscript received on September 11, 2016

Manuscript accepted on November 17, 2016

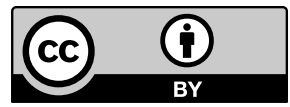

Motriz. The Journal of Physical Education. UNESP. Rio Claro, SP, Brazil - eISSN: 1980-6574 - under a license Creative Commons - Version 3.0 